\title{
Predictive factors for local recurrence and distant metastases following primary surgical treatment of phyllodes tumour of the breast
}

\author{
Ashwin Hebbar K. ${ }^{1 *}$, Sushil Kumar B. V. ${ }^{2}$, Shashidhar Kallapa ${ }^{3}$, Mohamad Arif ${ }^{4}$
}

\author{
${ }^{1}$ Assistant Professor, ${ }^{2}$ Professor, ${ }^{4}$ Associate Professor, Department of General Surgery, Shimoga Institute of Medical \\ Sciences, Shimoga, Karnataka, India \\ ${ }^{3}$ Associate Professor, Department of Surgical Oncology, Karnataka Institute of Medical Sciences, Hubli, Karnataka \\ State, India
}

Received: 20 March 2016

Accepted: 10 May 2016

\author{
*Correspondence: \\ Dr. Ashwin Hebbar K, \\ E-mail: hebbar2000@yahoo.com
}

Copyright: (c) the author(s), publisher and licensee Medip Academy. This is an open-access article distributed under the terms of the Creative Commons Attribution Non-Commercial License, which permits unrestricted non-commercial use, distribution, and reproduction in any medium, provided the original work is properly cited.

\begin{abstract}
Background: Phyllodes tumours are rare breast tumours that, like fibro adenomas, contain 2 types of breast tissue: stromal (connective) tissue and glandular (lobule and duct) tissue. They are most common in women in their 30 s and $40 \mathrm{~s}$, but they may also be found in women of any age. They constitute $1 \%$ of mammary tumours and $2-3 \%$ of fibro epithelial tumours of the breast. The objective of this study was to characterize the risk and predict factors for local and distant recurrence in patients primarily treated with surgery. And whether a subgroup could be identified in whom adjuvant treatment would be needed.

Methods: Data from 57 patients treated for phyllodes tumours between January 2008 to December 2012 (5 years) were reviewed retrospectively. Data recorded included age, menopausal status, diagnostic methods, treatment given, histopathology, recurrence and follow up. Preoperative diagnosis was done with fine needle aspiration cytology (FNAC). Work-up with - chest X-ray (CXR) and ultrasound (USG) abdomen. Patient characteristics, pathologic variables and surgical procedures were investigated as predictors of local recurrence and distant metastasis.

Results: Age of patients ranged from 30-60 years with a mean of 46 years. 24 patients were pre and 33 were postmenopausal. All patients presented with a self-detected breast lump. Right breast was involved in 33 patients and left in 24 patients. Central quadrant was most commonly involved (24 patients). 6 of our patients had clinically enlarged auxiliary nodes (FNAC - reactive). We performed simple mastectomy and wide local excision of the tumour. Histopathologically, 6 were benign, 24 were borderline and malignant were 27. Depending on histopathological report adjuvant treatment were given.

Conclusions: Preoperative diagnosis of phyllodes tumour of the breast is very crucial. The type of surgery, histological type, stromal overgrowth, cellular atypia and mitotic count were important factors in predicting local recurrence. The surgical margin, histological type, stromal overgrowth, cellular atypia and mitotic count were important factors in predicting distant metastasis. To prevent recurrence, wide local excision with at least $1 \mathrm{~cm}$ margin is preferred for benign and borderline types. Malignant types should be treated with simple mastectomy.
\end{abstract}

Keywords: Cystosarcoma phyllodes, Phyllodes tumour, Breast lump

\section{INTRODUCTION}

Phyllodes tumours are rare breast tumours that, like fibro adenomas, contain 2 types of breast tissue: connective tissue elements like stroma with high stromal cellularity and epithelial elements like glandular (lobule and duct) tissue. They are most common in middle aged women in their 30s and 40s, but they may be found in women of any age. They constitute less than $1 \%$ of mammary tumours and $2-3 \%$ of fibro epithelial tumours of the 
breast. In 1838 Johannes Muller coined the term "cystosarcoma phyllodes" for its leaf like and fleshy gross appearance. The term cystosarcoma is a misnomer since it is usually benign and metastasis is rare. It is therefore preferred that the term phyllodes tumour is used instead of cystosarcoma phyllodes. Other terms used for this tumour are pseudosarcomatous fibradenoma, giant intracanalicular fibradenoma and Brodie's serocystic disease. In 1981 WHO adopted the term phyllodes tumour. WHO come up with classification in 2003 for phyllodes tumour of the breast as benign, borderline and malignant varieties.

Surgery remains the mainstay of treatment for phyllodes tumour of the breast but extent of surgical approach continues to evolve extending from simple excision, wide local excision, and simple mastectomy to radical mastectomy. Among these, the most important factor may be the surgical margin. Therefore, a goal of surgical treatment is to ensure a negative surgical margin after excision. ${ }^{1}$ Adjuvant treatment for these remains controversial.

Recurring theme of this phyllodes tumour of the breast is the under diagnoses by pathologist and under treatment by surgeons. Hence accurate preoperative diagnosis and correct surgical planning is crucial. However, the preoperative diagnostic accuracy of phyllodes tumour is often poor because mammary phyllodes tumours are rare and their clinical, imaging, cytology and histology characteristics are similar to those of fibro adenomatous breast tumour, which has a high incidence. ${ }^{2,3}$ In addition, they theorized that the fibro adenoma was a possible precursor or progenitor lesion of the phyllodes tumour, an apparent possibility which, to date, cannot be completely ruled out in some cases. ${ }^{4,5}$ There was no change in dilemma in the last 180 years since it was described. The local recurrence is $5-30 \%$ and distant metastasis is 25 $40 \%$ especially in malignant variety.

Various patient characteristics, pathologic variables and surgical procedures are the predictors of local recurrence and distant metastasis. ${ }^{1}$ Age, tumour size, laterality and menopausal status were not of predictive value. Surgical approach, surgical margin, histiotype, mitotic count, and stromal overgrowth have been reported as predictive factors. ${ }^{5}$ Metastasis is rare and mainly haematogenous to lungs and bones. ${ }^{6}$ Lymphatic involvement is infrequent. ${ }^{7,8}$ Recent research interest has focused on the use of immunohistochemical markers, flow cytometry and other techniques to elucidate the cellular basis of tumour behaviour and for the development of additional prognostic indicators. ${ }^{9,10}$ Rarity of this phyllodes tumour precludes prospective studies. Again study on Indian patients has been a rarity.

The objective of this study was to characterize the risk and predict factors for local and distant recurrence in patients primarily treated with surgery. And whether a subgroup could be identified in whom adjuvant treatment would be needed.

\section{METHODS}

Data from 57 patients treated for phyllodes tumours between January 2008 to December 2012 (5 years) were reviewed retrospectively. Patient's data recorded included age, menopausal status, diagnostic methods, treatment given, histopathology, recurrence and follow up. Preoperative diagnosis was done with fine needle aspiration cytology (FNAC). Further work-up was done with chest X-ray (CXR) and ultrasonography (USG) of the abdomen.

\section{Methods of collection of the data}

All women with palpable breast lump of any age attending our hospital during that period and treated with wide local excision or mastectomy and histopathology reported as phyllodes tumour were retrospectively analyzed and included in the study.

In outpatient department a detailed history and thorough physical examination of the patient having palpable breast lump was carried out and entered in the proforma. The patient was informed about the procedure and informed consent was obtained from the patient before subjecting to fine needle aspiration cytology of the breast lump and bilateral mammography (Triple assessment). The standard procedure for fine needle aspiration cytology was followed, making use of a $10 \mathrm{ml}$ syringe bearing a 22-gauze needle (external diameter of $0.6 \mathrm{~mm}$ ) or rarely even 23 and 24 gauze needles. The fine needle aspiration cytology specimen was expressed on to a glass slide. It was then immersed in a fixative $95 \%$ methyl alcohol. The slides were stained with papanicolaou or giemsa stain. The interpretation of the slide was made by the same cytopathologyst.

The clinicocytological diagnosis was based upon palpation of the mass, degree of resistance at the aspiration biopsy, combined with microscopic examination of the aspirated cells. BIRADS (breast imaging radiology and data system) grading system for mammography was followed. The WHO classification (2003) of phyllodes tumour of the breast as benign, borderline and malignant was followed. Since the fine needle aspiration was done for palpable tumour, ultrasound guidance was not followed and repeat fine needle aspiration was carried out before open/excision biopsy if the pathologist reports the cytology slide as "inadequate". True cut biopsy was as such not followed. The patients were informed about the cytological diagnosis. If the lumps on the cytological examination were reported as malignant phyllodes tumour, benign or borderline tumour of size more than $5 \mathrm{cms}$ where breast conservation was not possible and recurrent phyllodes tumour simple mastectomy was performed. 
Rest of cases of benign and borderline phyllodes tumour wide local excision was done. The specimen was sent for the histopathological confirmation of the diagnosis.

Patient characteristics, pathologic variables and surgical procedures, margin status, adjuvant treatment given were retrospectively analyzed and investigated as predictors of local recurrence and distant metastasis.

\section{RESULTS}

All patients presented with self-detected breast lump who were subjected to fine needle aspiration cytology and definitive surgery later (wide local excision/simple mastectomy) and final histopathology as phyllodes tumour were retrospectively analyzed.

Thus data from 57 patients treated for phyllodes tumours between January 2008 to December 2012 (5 years) were reviewed retrospectively. Age of the patients in our study ranged from 30-60 years with a mean of 46 years. 24 patients were premenopausal and 33 were postmenopausal. Right breast was involved in 33 patients and left in 24 patients. Central quadrant was most commonly involved (24 patients). 6 of our patients had clinically enlarged axillary lymph nodes (FNAC-reactive).

Table 1: The result of fine needle aspiration cytology (FNAC) which was followed preoperatively in our study was as follows.

\begin{tabular}{|ll|}
\hline Preoperative Diagnosis & No. of patients \\
\hline Phyllodes tumour & 42 \\
\hline Fibrodenoma & 3 \\
\hline Spindle cell neoplasm & 9 \\
\hline Benign breast disease probably phyllodes & 3 \\
\hline
\end{tabular}

Mean tumour size was $8.6 \mathrm{cms}$. 12 patients had less than $5 \mathrm{cms}$ size and 45 patients had more than $5 \mathrm{cms}$ size tumour. This size of presentation is often larger than for fibro adenoma, although increased breast awareness and impact of screening has resulted in trend towards presentation at smaller tumour size. The WHO classification (2003) of phyllodes tumour of the breast as benign, borderline and malignant was followed. Histopathological criteria to diagnose benign, borderline and malignant phyllodes tumour were as follows.

Table 2: Histopathological criteria to diagnose benign, borderline and malignant phyllodes tumour.

\begin{tabular}{|lll|l|}
\hline Variables & Benign & Borderline & Malignant \\
\hline Mitosis per hpf & $0-4$ & $5-9$ & $>/=10$ \\
\hline Tumour necrosis & Absent & Absent & Present \\
\hline Cellular atypia & Mild & Moderate & Severe \\
\hline Stromal overgrowth & Mild & moderate & Severe \\
\hline
\end{tabular}

57 cases in our study the histopathology report was as follows.
Simple mastectomy was done for 36 patients and wide local excision was followed for 21 patients.

Table 3: Final histopathology report of phyllodes tumour.

\begin{tabular}{|ll|}
\hline Histopathology & No. of patients \\
\hline Benign & 6 \\
\hline Borderline & 24 \\
\hline Malignant & 27 \\
\hline
\end{tabular}

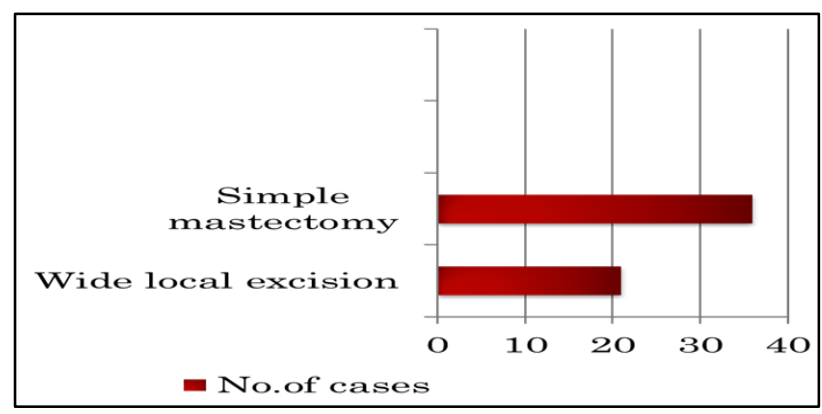

Figure 1: Surgical procedures that followed.

Adjuvant treatment in the form of radiotherapy was given for 18 cases, mainly for malignant phyllodes tumour.

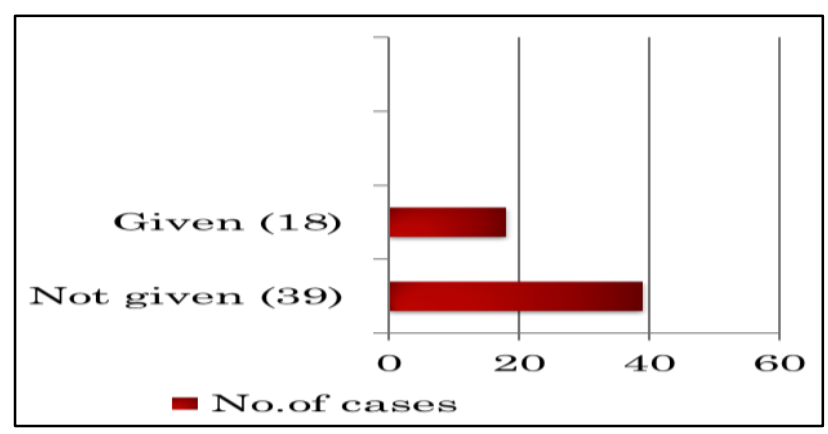

Figure 2: Adjuvant treatment RT.

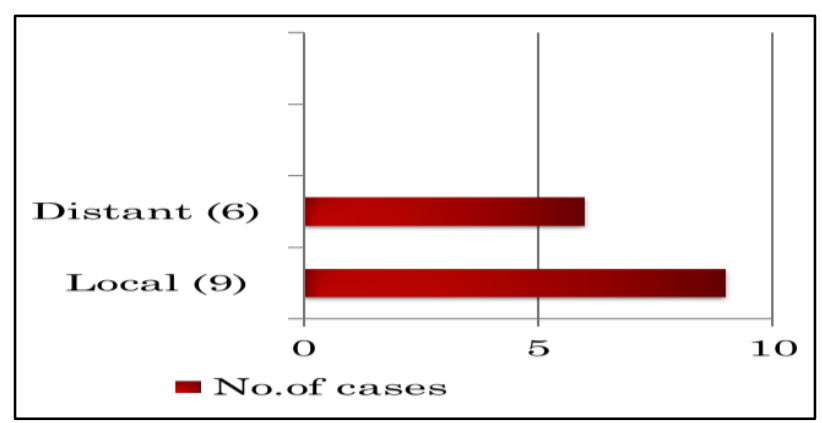

Figure 3: Recurrence status-local/distant.

Patient characteristics, pathologic variables and surgical procedures, margin status, adjuvant treatment given were retrospectively analyzed and investigated as predictors of local recurrence and distant metastasis. 
About 57 cases treated with surgical means (wide local excision and simple mastectomy) 15 patients had recurrence. 6 patients had distant recurrence whereas 9 patients had local recurrence.

Certain predictive factors were assessed for the development of local recurrence. Simple mastectomy (36 patients) was associated with recurrence in 3 patients, whereas wide local excision (21 patients) has got recurrence in 6 patients. Negative margin was associated with recurrence in 1 patients whereas negative but close $(<1 \mathrm{~cm})$ margin was associated with recurrence in 8 patients. Adjuvant treatment with radiation therapy (RT) (18 patients) was associated with local recurrence in 6 patients whereas no RT given (39 patients) was associated with recurrence in only 3 patients. This could be explained with the delivery of RT in only malignant variant of phyllodes tumours which were already aggressive in nature.

Table 4: Predictive factors for local recurrence in phyllodes tumour of the breast.

\begin{tabular}{|c|c|c|}
\hline Predictive factor & $\begin{array}{l}\text { Study group } \\
(\mathrm{No}=57)\end{array}$ & $\begin{array}{l}\text { Local recurrence } \\
(15.7 \%)(\mathrm{No}=9)\end{array}$ \\
\hline \multicolumn{3}{|l|}{ Type of surgery } \\
\hline WLE & 21 & 6 \\
\hline Mastectomy & 36 & 3 \\
\hline \multicolumn{3}{|l|}{ Margin status } \\
\hline -ve & 36 & 1 \\
\hline -ve but close $(<1 \mathrm{~cm})$ & 21 & 8 \\
\hline \multicolumn{3}{|l|}{ Adjuvant RT } \\
\hline Given & 18 & 6 \\
\hline Not given & 39 & 3 \\
\hline \multicolumn{3}{|l|}{ Histiotype } \\
\hline Benign & 6 & 1 \\
\hline Borderline & 24 & 2 \\
\hline Malignant & 27 & 6 \\
\hline \multicolumn{3}{|l|}{ Stromal overgrowth } \\
\hline Mild & 12 & 2 \\
\hline Moderate & 15 & 3 \\
\hline Severe & 30 & 4 \\
\hline \multicolumn{3}{|l|}{ Cellular atypia } \\
\hline Mild & 12 & 1 \\
\hline Moderate & 14 & 3 \\
\hline Severe & 31 & 5 \\
\hline \multicolumn{3}{|l|}{ Tumour necrosis } \\
\hline Present & 21 & 4 \\
\hline Absent & 36 & 5 \\
\hline \multicolumn{3}{|c|}{ Mitotic activity per HPF } \\
\hline$</=4$ & 15 & 2 \\
\hline $4-9$ & 18 & 1 \\
\hline$>/=10$ & 24 & 6 \\
\hline
\end{tabular}

Histologic variant associated with recurrence rate were also studied. Benign variant (6) was associated with recurrence in 1 patient, borderline variant (24) was associated with recurrence in 2 patients and malignant variant (27) was associated with recurrence in 6 patients. Severe stromal overgrowth (30 patients) was associated with local recurrence in 4 patients. Moderate stromal overgrowth (15) was associated with local recurrence in 3 patients. Mild stromal overgrowth (12) was associated with recurrence in 2 patients only. Mild cellular atypia (12 patients) was associated with local recurrence in 1 patient. Moderate cellular atypia (14) was associated with local recurrence in 3 patients and severe cellular atypia (31) was associated with recurrence in 5 patients. Relation of mitotic activity to local recurrence was also noted. Mitotic activity of more than 10 per high power field (HPF) (24 patients) was associated with local recurrence in 6 patients. Mitotic activity of 5-9 per high power field (18) was associated with local recurrence in 1 patient whereas mitotic activity less than 5 per high power field (15) was associated with local recurrence in 2 patients.

Distant recurrence or metastasis is rare in phyllodes tumour and mainly in malignant variety by haematogenous routs to lungs and bones. ${ }^{6}$ Like local recurrence certain predictive factors for the development of distant recurrence were also assessed. 6 of our patients, mostly malignant had distant recurrence all of them to the pulmonary metastasis. In type of surgery simple mastectomy (36 patients) was associated with distant recurrence in 5 patients, whereas wide local excision (21 patients) has got distant recurrence in 1 patient obviously for the reason simple mastectomy did for malignant and larger lesions. Negative margin as well as negative but close $(<1 \mathrm{~cm})$ margin was associated with distant recurrence in 3 patients each. Adjuvant treatment of RT (18 patients) was associated with distant recurrence in 4 patients whereas no RT given (39 patients) was associated with recurrence in only 2 patients. This again could be explained with the delivery of RT in only malignant variant of phyllodes tumours which were already aggressive in nature.

Histologic variant associated with distant recurrence/rates were also studied. Benign variant (6) was associated with no distant recurrence, borderline variant (24) was associated with distant recurrence in 1 patients and malignant variant (27) was associated with distant recurrence in 1 patient. Severe stromal overgrowth (30 patients) was associated with distant recurrence in 3 patients. Moderate stromal overgrowth (15) was associated with distant recurrence in 1 patient. Mild stromal overgrowth (12) was associated with recurrence in 2 patients only. Mild cellular atypia (12 patients) as well as moderate cellular atypia (14) was associated with distant recurrence in 1 patient each and severe cellular atypia (31) was associated with recurrence in 4 patients. Relation of mitotic activity to local recurrence was also noted. Mitotic activity of more than 10 per high power field (HPF) (24 patients) was associated with distant recurrence in 3 patients. Mitotic activity of 5-9 per high power field (18) was associated with local recurrence in 2 patient whereas mitotic activity less than 5 per high 
power field (15) was associated with local recurrence in 1 patients.

Follow up period in our study varies from 10-48 months (mean 16.3 months). During follow up period all patients were subjected for detail clinical examination, ultrasonography of abdomen and chest x-ray especially for borderline and malignant phyllodes tumours.

Table 5: Predictive factors for distant recurrence/metastasis in phyllodes tumour of the breast.

\begin{tabular}{|c|c|c|}
\hline Predictive factor & $\begin{array}{l}\text { Study group } \\
(\mathrm{No}=57)\end{array}$ & $\begin{array}{l}\text { Local recurrence } \\
(15.7 \%)(\mathrm{No}=9)\end{array}$ \\
\hline \multicolumn{3}{|l|}{ Type of surgery } \\
\hline WLE & 21 & 1 \\
\hline Mastectomy & 36 & 5 \\
\hline \multicolumn{3}{|l|}{ Margin status } \\
\hline -ve & 36 & 3 \\
\hline -ve but close $(<1 \mathrm{~cm})$ & 21 & 3 \\
\hline \multicolumn{3}{|l|}{ Adjuvant RT } \\
\hline Given & 18 & 4 \\
\hline Not given & 39 & 2 \\
\hline \multicolumn{3}{|l|}{ Histiotype } \\
\hline Benign & 6 & 0 \\
\hline Borderline & 24 & 1 \\
\hline Malignant & 27 & 5 \\
\hline \multicolumn{3}{|c|}{ Stromal overgrowth } \\
\hline Mild & 12 & 2 \\
\hline Moderate & 15 & 1 \\
\hline Severe & 30 & 3 \\
\hline \multicolumn{3}{|l|}{ Cellular atypia } \\
\hline Mild & 12 & 1 \\
\hline Moderate & 14 & 1 \\
\hline Severe & 31 & 4 \\
\hline \multicolumn{3}{|l|}{ Tumour necrosis } \\
\hline Present & 21 & 2 \\
\hline Absent & 36 & 4 \\
\hline \multicolumn{3}{|c|}{ Mitotic activity per hpf } \\
\hline$</=4$ & 15 & 1 \\
\hline $4-9$ & 18 & 2 \\
\hline$>/=10$ & 24 & 3 \\
\hline
\end{tabular}

High risk for local recurrence includes wide local excision compared to simple mastectomy, malignant histology, severe stromal overgrowth, severe cellular atypia and mitotic count more than 10 per high power field.

High risk for distant metastasis include malignant histology, close surgical margin, severe stromal overgrowth, severe cellular atypia and mitosis count more than 10 per high power field. Malignant histology, severe stromal overgrowth, severs cellular atypia and mitotic count more than 10 per high power field were predictive factor for both.

\section{DISCUSSION}

Phyllodes tumours are rare, accounting for less than $1 \%$ of all cases of mammary tumours. These are biphasic breast tumours occurring usually in adult females and are composed of benign epithelial component and a cellular spindle cell stroma forming leaf like structure. The biological features of these tumours include latent malignancy and the tumours show various clinical findings. Since phyllodes tumours tend to recur at the local site, local treatment requires wide excision to ensure inclusion of the tumour margin. Mastectomy is the most reliable procedure with regard to local control, but breastconservation surgery is currently selected in most cases (excluding cases with very large tumours) for aesthetic reasons. ${ }^{1}$

The frequency of local recurrence has varied from 8 to $46 \%$ in previous reports, and age, tumour size, surgical approach, mitotic activity, stromal overgrowth and surgical margin have been reported as prognosispredictive factors related to local recurrence. . $^{111-13}$

Mangi et al. reported that the frequency of local recurrence was correlated with the status of the surgical margin, and the incidence of local recurrence was very low in cases with $1 \mathrm{~cm}$ or wider margins 14 . In our study 9 patients out of 57 had local recurrence (15.7\%). 36 patients had clear negative margin in that recurrence was in only one patient (2.7\%). 21 patients had negative but close margin $(<1 \mathrm{~cm})$, among them the recurrence was in 8 patients $(38 \%)$. This clearly indicates that the wide local excision with close margin is an important predictive factor for local recurrence. To achieve excision ensuring a negative margin, the extent to which the phyllodes tumour was diagnosed before surgery is very significant. In our study phyllodes tumour was diagnosed preoperatively in 42 patients out of $57(73.68 \%)$ and 21 patients had negative but close margin $(<1 \mathrm{~cm})$, among them the recurrence was in 8 patients $(38 \%)$. This high rate of local recurrence was due to low rate of preoperative diagnosis and hence less achievement of negative margin. The preoperative diagnosis of phyllodes tumour may be increased by more frequent application of core needle biopsy and ultrasound guided mammotome biopsy. ${ }^{1}$

Many reports have recommended immediate excision to ensure a negative surgical margin in stump-positive cases because of the high local recurrence rate, whereas a 'wait-and-watch' policy has been proposed as acceptable for benign tumours because the local recurrence rate of benign and borderline phyllodes tumours is lower than that of malignant cases. In our study we also adopted 'wait-and-watch' policy of benign and borderline phyllodes tumour which account for 30 cases and most of the malignant tumours (27) and tumours more than $5 \mathrm{cms}$ were undergone mastectomy (36). 
Stromal overgrowth was a significant prognosis and predictive factor in close margin cases. In our study out of 9 cases of local recurrence severe stromal overgrowth was associated with local recurrence in 4 cases and moderate stromal overgrowth was associated with local recurrence in 3 cases. In the similar way malignant variant of phylloid tumour was associated with local recurrence in 6 cases, borderline variant was associated with local recurrence in 2 cases and benign variant was associated with local recurrence in 1 cases. After analyzing all these we recommend ensuring inclusion of the tumour margin by re-excision in cases of phyllodes tumour with a positive surgical margin with sever stromal overgrowth and malignant pathological variant. ${ }^{1,3}$

The role of adjuvant therapy in the form of radiation for phyllodes tumour remains unclear, since clinical data supporting the use of adjuvant radiation is again based on anecdotal case reports, rather than on large patient series. ${ }^{15,16}$ In our study of 27 cases of malignant phyllodes tumour radiation therapy was given in only 18 cases and local recurrence was observed in 6 cases $(33 \%)$. Radiation therapy was not given in 39 cases of phyllodes tumours most of them are benign and borderline (30) and some malignant variant which have undergone simple mastectomy with wide tumour clear margin and recurrence in these cases were only $3(7.6 \%)$. No series had shown radiation therapy to be of benefit in the primary treatment of phyllodes tumours. Even the M.D. Anderson series did not support the use of adjuvant radiation therapy for patients with malignant variant of phyllodes tumour with adequately resected margins. ${ }^{17}$ Role of adjuvant radiation therapy in patients with malignant phyllodes tumour with involved margin needs to be studied in larger prospective series.

Distant metastasis in phyllodes tumour mainly malignant variant had been reported up to $17 \%$ in various series. ${ }^{1,11-}$ ${ }^{13}$ In our series of 57 patients 6 patients had distant recurrence all of them to pulmonary site, observed over a mean follow-up period of 16.3 months. In patients with distant recurrence most of them were malignant phyllodes (5) tumour and 1 case was of borderline variant. Again distant recurrence was more common in patients with severe stromal overgrowth (3 cases) compared to moderate and mild. Severe cellular atypia (4 cases in our study) was more predictive factors for malignancy and hence for distant metastasis. The presence of tumour necrosis (4 cases in our study) was in the same way predictor of malignancy and distant metastasis. Mitotic activity more than 10 per high power field was also associated with distant recurrence. Adjuvant radiotherapy was given in 18 patients and among them distant metastasis was seen in 4 patients $(22 \%)$. Role of local radiotherapy in prevention of distant metastasis was not clear. ${ }^{18,19}$ Role of adjuvant chemotherapy was not evaluated in our study. However is was evident from other studies that wide local excision with adequate margins or mastectomy, was appropriate surgical treatment and adjuvant treatment (chemo- radiotherapy) for malignant tumours decreases recurrence rate and improves disease free survival. ${ }^{6,15}$

\section{CONCLUSION}

Preoperative diagnosis of phyllodes tumour of the breast is most crucial (42 patients in our study) which helps in proper planning of the surgical treatment as it prevent unnecessary re-surgery. The type of surgery, histiotype, stromal overgrowth, cellular atypia and mitotic count were important factors in predicting local recurrence. The surgical margin, histiotype, stromal overgrowth, cellular atypia and mitotic count were important factors in predicting distant metastasis.

A wide local excision with at least $1 \mathrm{~cm}$. margin is preferred for benign and borderline types. Malignant variety of phyllodes tumour of the breast should be treated with simple mastectomy. Since phyllodes tumour of the breast is a type of sarcoma where haematogenous spread is more common routine axillary dissection is not required. Adjuvant radiotherapy (RT) is indicated in malignant variety and in tumour with less than $1 \mathrm{~cm}$. margin in borderline variety of phyllodes tumour to prevent local recurrence. Local radiotherapy does not have any role to play in prevention of distant metastasis. Role of adjuvant chemotherapy was not evaluated in our study.

The limitations of our study included the small sample size, single institution experience and retrospective data which is not appropriate for accurately assessing the efficacy of any given treatment. A larger sample with multi-institutional and prospective data collection is required for defining proper protocol for phyllodes tumour of the breast and assessing risk factors for local recurrence and distant metastasis.

\section{ACKNOWLEDGEMENTS}

The Author would express deep sense of gratitude to professor of surgical oncology Dr. Krishna Murthy S., whose guidance and encouragement all throughout his periodic assessment and specific corrections, coupled with his rich knowledge and deep interest in the topic was our constant source of inspiration leading to the success of this study.

\section{Funding: No funding sources \\ Conflict of interest: None declared \\ Ethical approval: Not required}

\section{REFERENCES}

1. Taira N, Takabatake D, Aogi K. Phyllodes tumour of the breast: stromal overgrowth and histological classification are useful prognosis-predictive factors for local recurrence in patients with a positive surgical margin. JPN Journal of Clinical Oncology. 2007;37(10):730-6. 
2. Yilmaz E, Sal S, Lebe B. Differentiation of phyllodes tumours versus fibroadenomas. Acta Radiol. 2002;43:34-9.

3. Liberman L, Bonaccio E, Hamele-Bena D, Abramson AF, Cohen MA, Dershaw DD. Benign and malignant phyllodes tumours: mammographic and sonographic findings. Radiology. 1996;198:1214.

4. Noguchi S, Yokouchi H, Aihara T, Motomura K, Inaji $\mathrm{H}$, Imaoka $\mathrm{S}$, et al. Progression of fibroadenoma to phyllodes tumour demonstrated by clonal analysis. Cancer. 1995;76:1779-85.

5. Jara-Lazaro AR, Tan PH. Molecular pathogenesis of progression and recurrence in phyllodes tumours. American Journal of Translational Research. 2009;1(1):23-34.

6. Khatoon S, Arif M. Clinical pattern and management outcome of phyllodes tumour of breast. Pakistan Journal of Surgery (International). 2008:13(1):16-8.

7. Stranzl H, Peintinger F, Hackl A. Phyllodes tumour: an unexpected tumour of the breast. A report on six patients. Strahlenther Onkol. 2004;180:148-51.

8. Guerrero MA, Ballard BR, Grau AM. Malignant phyllodes tumour of the breast: review of the literature and case report of stromal overgrowth. Surgical Oncology. 2003;12:27-37.

9. Jacklin RK, Ridgway PF, Ziprin P, Healy V, Hadjiminas D, Darzi A. Optimising preoperative diagnosis in phyllodes tumour of the breast. Journal of clinical pathology. J Clin Pathol. 2006;59(5):4549.

10. Palko MJ, Wang SE, Shackney SE, Cottington EM, Levitt SB, Hartsock RJ. Flow cytometric S fraction as a predictor of clinical outcome in cystosarcoma phyllodes. Arch Pathol Lab Med. 1990;114:949-52.

11. Reinfuss M, Mitus J, Duda K, Stelmach A, Rys J, Smolak K. The treatment and prognosis of patients with phyllodes tumor of the breast: an analysis of 170 cases. Cancer. 1996;77:910-2.

12. Pietruszka M, Barnes L. Cystosarcoma phyllodes: a clinicopathologic analysis of 42 cases. Cancer. 1978;41:1974-83.

13. Hines JR, Murad TM, Beal JM. Prognostic indicators in cystosarcoma phylloides. American Journal of Surgery. 1987;153:276-80.

14. Mangi AA, Smith BL, Gadd MA, Tanabe KK, Ott MJ, Souba WW. Surgical management of phyllodes tumours. Arch Surg. 1999;134:487-92.

15. Pandey M, Mathew A, Kattoor J, Abraham EK, Mathew BS, Rajan B, et al. Malignant phyllodes tumour. Breast J. 2001;7:411-6.

16. Eich PD, Diederich S, Eich HT, Micke O, Wagner W. Diagnostic radiation oncology: malignant cystosarcoma phylloides. Strahlenther Onkol. 2000;176:192-5.

17. Chaney AW, Pollack A, McNeese MD, Zagars GK, Pisters PW, Pollock RE, et al. Primary treatment of cystosarcoma phyllodes of the breast. Cancer. 2000;89:1502-11.

18. DeVita, Hellman, Rosenberg. Cancer- Principles and Practice of Oncology. $9^{\text {th }}$ edition. New York, Lippincott Williams and Wilkins; 2011:1438.

19. Abdalla HM, Sakr MA. Predictive factors for local recurrence and distant metastases following primary surgical treatment of phyllodes tumour of breast. J Egypt Natl Canc Inst. 2006;18(2):125-33.

Cite this article as: Hebbar AK, Kumar SBV, Kallapa S, Arif M. Predictive factors for local recurrence and distant metastases following primary surgical treatment of phyllodes tumour of the breast. Int Surg J 2016;3:1379-85. 